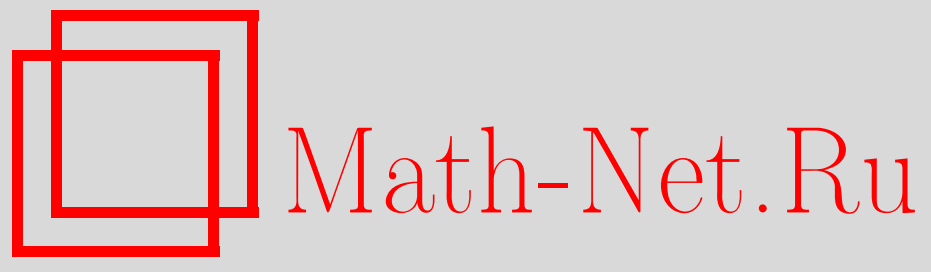

В. Э. Адлер, А. Б. Шабат, Одевающая цепочка для акустической спектральной задачи, ТМ $\Phi, 2006$, том 149, номер 1, 32-46

DOI: https://doi.org/10.4213/tmf3826

Использование Общероссийского математического портала Math-Net.Ru подразумевает, что вы прочитали и согласны с пользовательским соглашением http://www .mathnet.ru/rus/agreement

Параметры загрузки:

IP : 3.85 .183 .62

26 апреля 2023 г., $17: 36: 48$

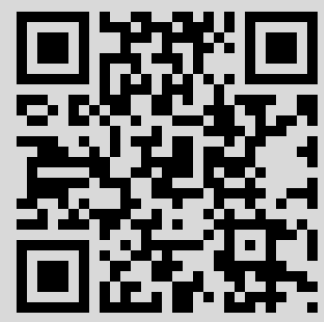




\section{ОДЕВАЮЩАЯ ЦЕПОЧКА ДЛЯ АКУСТИЧЕСКОЙ СПЕКТРАЛЬНОЙ ЗАДАЧИ}

Изучены итерации преобразования Дарбу для обобщенного оператора Шредингера. Рассмотрены приложения к уравнениям Дима и Камассы-Холма.

Ключевые слова: преобразование Дарбу, одевающая цепочка, уравнение Дима, уравнение Камассы-Холма.

\section{1. ВВЕДЕНИЕ}

В работе рассматривается обобщенное уравнение Шредингера

$$
\varphi_{y y}=\left(q(y)-\lambda r^{4}(y)\right) \varphi
$$

и ассоциированные с ним уравнения Дима [1] и Камассы-Холма [2]. Хотя это уравнение приводится к стандартному виду $r=1$ преобразованием Лиувилля, перенесение известных результатов является не вполне тривиальным из-за необходимости менять независимую переменную. Это приводит к известным трудностям как в самой постановке краевых задач, так и в построении их решений. В частности, все известные точные решения записываются не явно, а в параметрическом виде, причем многие из них имеют особенности. Среди работ, посвященных разработке теории рассеяния, отметим [3], [4, гл. 6.5], [5], [6]. Построению алгебро-геометрических, многосолитонных и пиконных решений посвящены работы [7] и др. Преобразования Дарбу-Беклунда рассматриваются в работах [8], но техника этих преобразований, на наш взгляд, пока еще недостаточно развита. Между тем для оператора Шредингера преобразование Дарбу зарекомендовало себя как эффективный инструмент построения точно решаемых потенциалов и явных решений уравнения Кортевегаде Фриза (КдФ) (см., например, [9]-[11] и многие другие работы). Продублировать имеющиеся результаты не всегда удается в силу объективных различий в постановке спектральных задач, но следует хотя бы воспроизвести основные идеи. Это мы и пытаемся сделать в представленной статье.

${ }^{*}$ Институт теоретической физики им. Л.Д. Ландау РАН, Черноголовка, Московская обл., Россия. E-mail: adler@itp.ac.ru, shabat@itp.ac.ru 
Наличие двух потенциалов дает возможность комбинировать преобразования Дарбу и Лиувилля. Это приводит к интересным последствиям и заслуживает всестороннего изучения. В данной работе ключевым является наблюдение, что одна из таких комбинаций устроена почти так же просто, как само преобразование Дарбу, но обслуживает калибровку $q=0$, а не $r=1$.

\section{2. ПРЕОБРАЗОВАНИЯ ЛИУВИЛЛЯ И ДАРБУ}

Цель данного раздела - ввести в рассмотрение преобразования, действующие на множестве обобщенных уравнений Шредингера

$$
\varphi_{y y}=\left(q(y)-\lambda r^{4}(y)\right) \varphi
$$

Классическое преобразование Лиувилля имеет общий вид

$$
\varphi_{y y}=U \varphi \quad \leftrightarrow \quad \widetilde{\varphi}_{\tilde{y} \tilde{y}}=\widetilde{U} \widetilde{\varphi}, \quad d \tilde{y}=a^{2} d y, \quad \widetilde{\varphi}=a \varphi, \quad \widetilde{U}=\frac{U}{a^{4}}+\frac{a_{\tilde{y} \tilde{y}}}{a}
$$

где $a(\tilde{y})$ - произвольная функция. Мы используем его частные случаи для приведения уравнения (1) к нескольким специальным формам. Начнем с преобразования, уничтожающего множитель $r$. Ввиду важности этой формы уравнения мы используем для нее свои обозначения.

УтвеРЖДЕНИЕ 1 (преобразование Лиувилля). Уравнение (1) приводится $к$ виду

$$
\psi_{x x}=(u(x)-\lambda) \psi
$$

заменой

$$
d x=r^{2} d y, \quad \psi=r \varphi, \quad u=\frac{q}{r^{4}}+\frac{r_{x x}}{r} .
$$

Другое классическое преобразование определяется по частному решению $\psi^{(\alpha)}$ уравнения (2) при $\lambda=\alpha$. Нетрудно пересчитать его и в общей калибровке (1).

УтвеРЖДЕниЕ 2 (преобразование Дарбу). Уравнение (2) сохраняет вид при преобразовании

$$
\hat{\psi}=\psi_{x}-f \psi, \quad f:=\frac{\psi_{x}^{(\alpha)}}{\psi^{(\alpha)}}, \quad f_{x}+f^{2}=u-\alpha, \quad \hat{u}=u-2 f_{x} .
$$

Уравнение (1) сохраняет вид при преобразовании

$$
\begin{gathered}
\widehat{\varphi}=\frac{1}{r^{2}}\left(\varphi_{y}-f \varphi\right), \quad f:=\frac{\varphi_{y}^{(\alpha)}}{\varphi^{(\alpha)}}, \quad f_{y}+f^{2}=q-\alpha r^{4}, \\
\hat{r}=r, \quad \hat{q}=q-2 f_{y}+\frac{4 r_{y}}{r} f+6 \frac{r_{y}^{2}}{r^{2}}-2 \frac{r_{y y}}{r} .
\end{gathered}
$$

Теперь рассмотрим другую каноническую форму уравнения (1):

$$
\varphi_{y y}=-\lambda r^{4}(y) \varphi
$$

2 Теоретическая и математическая физика, т. 149, № 1, 2006 г. 
Так называемая акустическая спектральная задача (5) отвечает потенциалу $r$, отделенному от 0 и достаточно быстро сходящемуся к 1 при $|y| \rightarrow \infty$ [5]. Однако вначале мы не будем заботиться об аналитике и будем ссылаться на (5) просто как на акустическое уравнение. Очевидно, что оно получается из уравнения (2) обратным преобразованием Лиувилля, если выбрать $q=0$, а в качестве $r$ взять волновую функцию при $\lambda=0$ :

$$
d x=r^{2} d y, \quad \psi=r \varphi, \quad u=\frac{r_{x x}}{r} .
$$

Несложно показать, что произвол в выборе $r$ сводится к дробно-линейным заменам на множестве уравнений (5):

$$
\tilde{y}=\frac{c_{1} y+c_{2}}{c_{3} y+c_{4}}, \quad \tilde{\varphi}=\frac{1}{c_{3} y+c_{4}} \varphi, \quad \tilde{r}=\frac{c_{3} y+c_{4}}{\Delta^{1 / 2}} r, \quad \Delta=c_{1} c_{4}-c_{2} c_{3} .
$$

Пересчет преобразования Дарбу приводит к неожиданно простым формулам.

УтвЕРжДЕНИЕ 3. Уравнение (5) сохраняет вид при преобразовании

$$
\begin{gathered}
\widehat{\varphi}=\frac{\varphi_{y}}{p}-\varphi, \quad p:=\frac{\varphi_{y}^{(\alpha)}}{\varphi^{(\alpha)}}, \quad p_{y}+p^{2}=-\alpha r^{4}, \\
\hat{r}=\frac{p}{r}, \quad \hat{r}^{2} d \hat{y}=r^{2} d y .
\end{gathered}
$$

ДокАЗАТЕЛЬСтво. Применяя $D_{\hat{y}}=p^{2} r^{-4} D_{y}$ и исключая $p_{y}$ в силу уравнения Риккати, получаем сначала $\widehat{\varphi}_{\hat{y}}=\alpha \varphi_{y}-\lambda p \varphi$, а затем $\widehat{\varphi}_{\hat{y} \hat{y}}=-\lambda p^{4} r^{-4} \widehat{\varphi}$.

Чтобы проверить, что данное преобразование эквивалентно (4), применим замену (6) к $\varphi$ и $\widehat{\varphi}$. Учитывая соотношение $f=\psi_{x}^{(\alpha)} / \psi^{(\alpha)}=r_{x} / r+p / r^{2}$, получаем

$$
\hat{\psi}=\hat{r} \widehat{\varphi}=\frac{p}{r}\left(\frac{1}{p} \varphi_{y}-\varphi\right)=r \varphi_{x}-\frac{p}{r} \varphi=r\left(\frac{\psi}{r}\right)_{x}-\frac{p \psi}{r^{2}}=\psi_{x}-f \psi .
$$

Уравнение (5) рассматривается также на конечном интервале [3]. В этом случае можно перейти к спектральной задаче на всей оси

$$
\chi_{z z}=\left(1-\lambda R^{4}(z)\right) \chi
$$

при помощи следующего преобразования Лиувилля [5]:

$$
y=\operatorname{th} z, \quad \varphi=\chi \operatorname{sech} z, \quad r=R \operatorname{ch} z .
$$

При этом предполагается, что $r \in C^{\infty}([-1,1]), r>0, r(-1)=r(1)$. Эта калибровка важна потому, что с ней связано уравнение Камассы-Холма, которое обсуждается в разделе 5. Однако в этих переменных преобразование Дарбу выглядит достаточно неуклюже, и удобнее по-прежнему пользоваться формулами (8), пересчитывая ответ с помощью указанной замены.

В заключение приведем еще два простых автопреобразования акустического уравнения. 
УтВЕРЖДЕНИЕ 4. Вид уравнения (5) сохраняется при преобразованиях

$$
\begin{gathered}
\bar{\varphi}=\varphi_{y}, \quad \bar{r}=\frac{1}{r}, \quad d \bar{y}=r^{4} d y, \\
\tilde{\varphi}=\frac{\varphi}{\varphi^{(\alpha)}}, \quad \tilde{r}=r \varphi^{(\alpha)}, \quad\left(\varphi^{(\alpha)}\right)^{2} d \tilde{y}=d y, \quad \tilde{\lambda}=\lambda-\alpha .
\end{gathered}
$$

ДоКАЗАТЕЛьство. Для первого преобразования имеем

$$
\bar{\varphi}_{\bar{y}}=r^{-4} \varphi_{y y}=-\lambda \varphi, \quad \bar{\varphi}_{\bar{y} \bar{y}}=-\lambda r^{-4} \varphi_{y}=-\lambda \bar{r}^{4} \bar{\varphi} .
$$

Данное преобразование эквивалентно преобразованию Дарбу (4), порожденному функцией $\psi^{(0)}=r$. Действительно,

$$
\bar{\psi}=\bar{r} \bar{\varphi}=\frac{\varphi_{y}}{r}=r \varphi_{x}=r\left(\frac{\psi}{r}\right)_{x}=\psi_{x}-r_{x} \frac{\psi}{r} .
$$

Отметим, что формулы (8) отвечают выбору $\psi^{(0)}=(y+c) r=r \int r^{-2} d x+c r$.

Для второго преобразования имеем

$$
\begin{gathered}
\widetilde{\varphi}_{\tilde{y}}=\varphi_{y} \varphi^{(\alpha)}-\varphi \varphi_{y}^{(\alpha)}, \\
\widetilde{\varphi}_{\tilde{y} \tilde{y}}=\left(\varphi_{y y} \varphi^{(\alpha)}-\varphi \varphi_{y y}^{(\alpha)}\right)\left(\varphi^{(\alpha)}\right)^{2}=(\alpha-\lambda) r^{4} \varphi\left(\varphi^{(\alpha)}\right)^{3}=(\alpha-\lambda) \tilde{r}^{4} \widetilde{\varphi} .
\end{gathered}
$$

При замене (6) получаем

$$
\tilde{\psi}=\tilde{r} \tilde{\varphi}=\psi, \quad \tilde{u}=\frac{\tilde{r}_{x x}}{\tilde{r}}=\frac{r_{x x}}{r}+\frac{1}{\varphi^{(\alpha)}}\left(\frac{2 r_{x}}{r} \varphi_{x}^{(\alpha)}+\varphi_{x x}^{(\alpha)}\right)=u-\alpha,
$$

т.е. это преобразование соответствует сдвигу

$$
\tilde{\psi}=\psi, \quad \tilde{u}=u-\alpha, \quad \tilde{\lambda}=\lambda-\alpha
$$

в уравнении Шредингера.

\section{3. ОДЕВАЮЩИЕ ЦЕПОЧКИ}

При рассмотрении итераций преобразования Дарбу (8) удобно ввести дополнительное растяжение, полагая $\hat{r}_{n}=-\gamma_{n} r_{n+1}, \hat{y}_{n}=\gamma_{n}^{-2} y_{n+1}$. Вводя параметр $x$ по формуле $d x=r_{n}^{2} d y_{n}$ и исключая $p_{n}$ из соотношений $p_{n}=-\gamma_{n} r_{n+1} r_{n}, p_{n, y_{n}}+p_{n}^{2}=$ $-\alpha_{n} r_{n}^{4}$, получаем последовательность уравнений $\left(r_{n+1} r_{n}\right)_{x}=\gamma_{n} r_{n+1}^{2}+\left(\alpha_{n} / \gamma_{n}\right) r_{n}^{2}$, которая выглядит особенно симметричной при выборе $-\gamma_{n}^{2}=\alpha_{n}$.

При этом мы теряем преобразование Дарбу, отвечающее $\alpha=0$. Ясно, однако, что это преобразование действительно отличается от остальных, и его следует рассматривать отдельно. Это будет сделано в разделе 5. Итак, мы пришли к уравнениям

$$
\left(r_{n+1} r_{n}\right)_{x}=\gamma_{n}\left(r_{n+1}^{2}-r_{n}^{2}\right), \quad y_{n, x}=r_{n}^{-2} .
$$

Первое из них при замене $r_{n}=e^{g_{n}}$ принимает вид

$$
g_{n+1, x}+g_{n, x}=2 \gamma_{n} \operatorname{sh}\left(g_{n+1}-g_{n}\right)
$$


и задает $x$-часть преобразования Беклунда для потенциального модифицированного уравнения КдФ и уравнения sh-Гордон (см., например, [10])

$$
g_{t}=g_{x x x}-2 g_{x}^{3}, \quad g_{x \tau}=\operatorname{sh} 2 g .
$$

Дифференциально-разностные уравнения подобного вида мы будем называть одевающими иепочками. Этот термин был введен в работах [11], [12] применительно к уравнениям

$$
f_{n+1, x}+f_{n, x}=f_{n}^{2}-f_{n+1}^{2}+\alpha_{n}-\alpha_{n+1},
$$

описывающим итерации преобразования Дарбу (4). Как было показано в предыдущем разделе, преобразования (4) и (8) сопряжены преобразованием Лиувилля (6), следовательно, между цепочками (12) и (14) должна быть какая-то связь. Чтобы найти ее, введем потенциал $v$ по формулам

$$
2 v_{n, x}=u_{n}, \quad v_{n}-v_{n+1}=f_{n} .
$$

Для этих переменных выполняется хорошо известное свойство коммутативности преобразований Дарбу (см., например, [10]).

УтВеРЖДЕНИЕ 5. Пусть $v, v^{(\alpha)} u v^{(\beta)}$ связаны преобразованиями Дарбу

$$
v_{x}^{(\alpha)}+v_{x}=\left(v^{(\alpha)}-v\right)^{2}+\alpha, \quad v_{x}^{(\beta)}+v_{x}=\left(v^{(\beta)}-v\right)^{2}+\beta .
$$

Тогда величина

$$
v^{(\alpha, \beta)}=v-\frac{\alpha-\beta}{v^{(\alpha)}-v^{(\beta)}}
$$

связана с $v^{(\alpha)}$ u $v^{(\beta)}$ преобразованиями Дарбу

$$
v_{x}^{(\alpha, \beta)}+v_{x}^{(\beta)}=\left(v^{(\alpha, \beta)}-v^{(\beta)}\right)^{2}+\alpha, \quad v_{x}^{(\alpha, \beta)}+v_{x}^{(\alpha)}=\left(v^{(\alpha, \beta)}-v^{(\alpha)}\right)^{2}+\beta .
$$

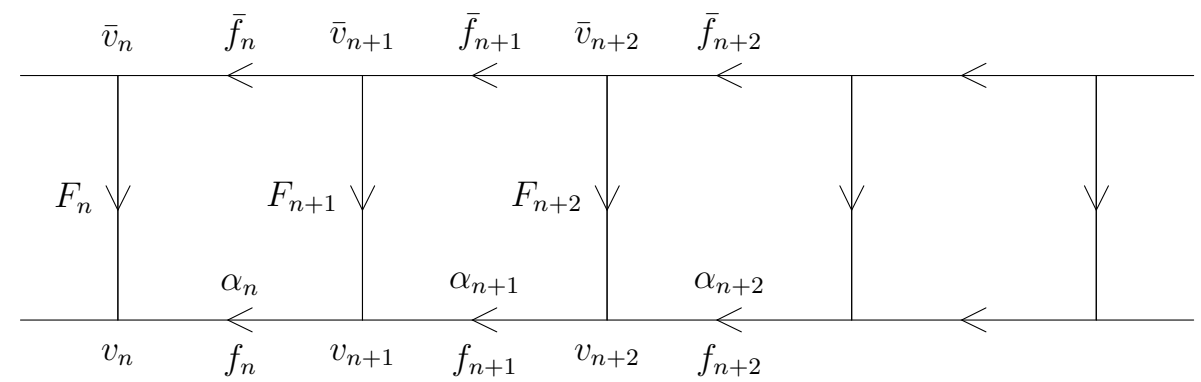

Рис. 1. Две копии одевающей цепочки.

Далее, рассмотрим одевающую цепочку для переменных $v_{n}$

$$
v_{n+1, x}+v_{n, x}=\left(v_{n+1}-v_{n}\right)^{2}+\alpha_{n}
$$

и ее копию для переменных $\bar{v}_{n}$, связанных преобразованием Дарбу с нулевым параметром:

$$
v_{n, x}+\bar{v}_{n, x}=\left(v_{n}-\bar{v}_{n}\right)^{2} .
$$


Совместность уравнений (16) с обеими копиями цепочки обеспечивается утверждением 5, причем выполняются соотношения

$$
\left(v_{n+1}-\bar{v}_{n}\right)\left(v_{n}-\bar{v}_{n+1}\right)=\alpha_{n} .
$$

Разности $f_{n}, \bar{f}_{n}$ отождествляются с ориентированными горизонтальными ребрами фигуры, изображенной на рис 1 . Несложно показать, что разности $F_{n}=v_{n}-\bar{v}_{n}$, отвечающие вертикальным ребрам, удовлетворяют цепочке

$$
F_{n+1, x}+F_{n, x}=\left(F_{n+1}-F_{n}\right) \sqrt{\left(F_{n+1}+F_{n}\right)^{2}-4 \alpha_{n}} .
$$

Действительно,

$$
\begin{aligned}
\left(F_{n+1}+F_{n}\right)_{x} & =\left(v_{n+1}+v_{n}-\bar{v}_{n+1}-\bar{v}_{n}\right)_{x}=\left(v_{n+1}-v_{n}\right)^{2}-\left(\bar{v}_{n+1}-\bar{v}_{n}\right)^{2}= \\
& =\left(v_{n+1}-v_{n}-\bar{v}_{n+1}+\bar{v}_{n}\right)\left(v_{n+1}-v_{n}+\bar{v}_{n+1}-\bar{v}_{n}\right)= \\
& =\left(F_{n+1}-F_{n}\right) \sqrt{\left(v_{n+1}+v_{n}-\bar{v}_{n+1}-\bar{v}_{n}\right)^{2}-4\left(v_{n+1}-\bar{v}_{n}\right)\left(v_{n}-\bar{v}_{n+1}\right)}= \\
& =\left(F_{n+1}-F_{n}\right) \sqrt{\left(F_{n+1}+F_{n}\right)^{2}-4 \alpha_{n}},
\end{aligned}
$$

где последнее равенство вытекает из формулы (17).

Цепочка (18) связана с (12) заменой $F_{n}=r_{n, x} / r_{n}$. Напомним, что оператор $D_{x}-$ $r_{x} / r$ определяет специальное преобразование Дарбу при $\alpha=0$ (см. доказательство утверждения 4).

Чтобы вернуться к цепочке (14), заметим, что соотношение (17) эквивалентно квадратному уравнению относительно $f_{n}$. Решая его, получаем подстановки в цепочку (14) и ее копию, отвечающую переменным $\bar{f}_{n}$.

УтвеРЖДЕНИЕ 6. Общие решения цепочек (14), (18) и (12) связаны подстановками

$$
\begin{aligned}
& F_{n}=\frac{r_{n, x}}{r_{n}}, \quad s_{n}:=\sqrt{\left(F_{n+1}+F_{n}\right)^{2}-4 \alpha_{n}}=\gamma_{n}\left(\frac{r_{n+1}}{r_{n}}+\frac{r_{n}}{r_{n+1}}\right), \quad \alpha_{n}=-\gamma_{n}^{2}, \\
& 2 f_{n}=F_{n}-F_{n+1}-s_{n}, \quad 2 \bar{f}_{n}=F_{n+1}-F_{n}-s_{n}, \\
& f_{n}=\frac{r_{n, x}-\gamma_{n} r_{n+1}}{r_{n}}, \quad \bar{f}_{n}=-\frac{r_{n, x}}{r_{n}}-\frac{\gamma_{n} r_{n}}{r_{n+1}} .
\end{aligned}
$$

До сих пор мы не обращали внимания на переменные $y_{n}$. Оказывается, для них можно выписать отдельную цепочку. Разделив первое уравнение цепочки (12) на $r_{n+1}^{2} r_{n}^{2}$ и интегрируя, получаем формулу (постоянные интегрирования здесь несущественны и убираются сдвигом $\left.y_{n} \rightarrow y_{n}+c_{n}\right)$

$$
\frac{1}{\gamma_{n} r_{n+1} r_{n}}=y_{n+1}-y_{n},
$$

которая означает, что для нахождения всех $y_{n}$ достаточно одной квадратуры. Эти уравнения можно переписать также в виде

$$
y_{n+1, x} y_{n, x}=\gamma_{n}^{2}\left(y_{n+1}-y_{n}\right)^{2} .
$$


Наконец, отметим, что замена $\bar{r}_{n}=1 / r_{n}$, порожденная преобразованием (11), эквивалентна обращению вертикальных стрелок на рис. 1 и приводит к цепочкам

$$
\left(\bar{r}_{n+1} \bar{r}_{n}\right)_{x}=\gamma_{n}\left(\bar{r}_{n+1}^{2}-\bar{r}_{n}^{2}\right), \quad \bar{y}_{n+1, x} \bar{y}_{n, x}=\gamma_{n}^{2}\left(\bar{y}_{n+1}-\bar{y}_{n}\right)^{2}, \quad \bar{y}_{n, x}=\bar{r}_{n}^{-2} \text {. }
$$

Для переменных $\bar{y}_{n}$ имеем рекуррентную формулу, аналогичную (20):

$$
\bar{y}_{n+1}-\bar{y}_{n}=\frac{r_{n+1} r_{n}}{\gamma_{n}} .
$$

\section{4. УРАВНЕНИЕ ДИМА}

Одевающие цепочки, приведенные в предыдущем разделе, относятся к весьма важному классу преобразований Беклунда вида

$$
u_{n+1, x}=b\left(u_{n, x}, u_{n}, u_{n+1}, \alpha_{n}\right),
$$

обслуживающему уравнения типа КдФ

$$
u_{t}=A\left(u_{x x x}, u_{x x}, u_{x}, u, x, \alpha\right)
$$

Например, цепочки (14) и (18) задают $x$-части преобразований Беклунда для модифицированных уравнений КдФ

$$
f_{t}=f_{x x x}-6\left(f^{2}+\alpha\right) f_{x}, \quad F_{t}=F_{x x x}-6 F^{2} F_{x},
$$

а цепочки (21) и (15) соответственно для уравнений

$$
y_{t}=y_{x x x}-\frac{3 y_{x x}^{2}}{2 y_{x}}, \quad v_{t}=v_{x x x}-6 v_{x}^{2} .
$$

Цепочка (13), как уже упоминалось, отвечает потенциальному модифицированному уравнению КдФ, или, для $r$,

$$
r_{t}=r_{x x x}-\frac{3 r_{x x} r_{x}}{r}
$$

Список легко продолжить, но при этом мы не выходим из подкласса уравнений вида

$$
u_{t}=u_{x x x}+a\left(u_{x x}, u_{x}, u, x, \alpha\right) .
$$

Действительно, совместность уравнений (23), (24) означает, что выполняется тождество

$$
D_{x}(A[n+1])=b_{u_{n, x}} D_{x}(A[n])+b_{u_{n}} A[n]+b_{u_{n+1}} A[n+1],
$$

где производные от $u_{n+1}$ исключены в силу (23). Равенство коэффициентов при $u_{n, x x x x}$ дает соотношение $A_{u_{x x x}}[n+1]=A_{u_{x x x}}[n]$. Величина $A_{u_{x x x}}^{-1 / 3}$ называется сепарантой уравнения (24). Если она зависит только от $x$, то уравнение приводится к виду (26). В противном случае цепочка (23) не выводит за пределы конечнопараметрического семейства решений обыкновенного дифференциального уравнения 
вида $A_{u_{x x x}}=A_{u_{x x x}}[0]=c(x)$. Ясно, что такое преобразование Беклунда не может считаться полноценным.

Тем не менее цепочка вида (23) все же может определять преобразование Беклунда для уравнения с переменной сепарантой, если ее расширить некоторым уравнением для независимой переменной, которую мы теперь будем обозначать $y_{n}$. При этом $x$ считается вспомогательным параметром. Цепочка (12) как раз дает пример такого расширения, отвечающий уравнению Дима для переменной $w=r^{-2}$ :

$$
w_{t}=w^{3} w_{y y y} .
$$

Действительно, это уравнение связано с (25) следующей композицией преобразования годографа и двух введений потенциала:

$$
\begin{array}{ccc}
x_{y}=\frac{1}{w}, \quad x_{t}=\frac{1}{2} w_{y}^{2}-w w_{y y} & y_{x}=\frac{1}{r^{2}}, \quad y_{t}=-\frac{2 r_{x x}}{r^{3}} \\
\Downarrow & \Downarrow \\
x_{t}=\frac{x_{y y y}}{x_{y}^{3}}-\frac{3 x_{y y}^{2}}{2 x_{y}^{4}} \quad \Leftrightarrow \quad y_{t}=y_{x x x}-\frac{3 y_{x x}^{2}}{2 y_{x}}
\end{array}
$$

(подобные замены несколько компактнее записываются как преобразования по решению). Соотношение $w=r^{-2}$ является просто следствием тождества $y_{x} x_{y}=1$.

ЗАмечание 1. Расширение цепочки может быть не единственным. Действительно, уравнение (25) допускает более общий потенциал:

$$
y_{x}=a r^{-2}+b r^{2}, \quad y_{t}=-2 a r_{x x} r^{-3}+2 b\left(r r_{x x}-2 r_{x}^{2}\right) .
$$

Это можно использовать для сохранения вещественности при $r \sim e^{i g}$, что отвечает уравнению sin-Гордон и модифицированному уравнению КдФ со знаком плюс перед нелинейным членом. В частности, цепочку

$$
g_{n+1, x}+g_{n, x}=2 \gamma_{n} \sin \left(g_{n+1}-g_{n}\right)
$$

можно расширить, положив $y_{n, x}=c+\sin 2 g_{n}$, где постоянная $c \geqslant 1$ добавлена, чтобы обеспечить однозначность замены $x \leftrightarrow y$ на всей оси. Это приводит к следующей последовательности преобразований:

$$
\begin{array}{cc}
w_{t}=w^{3} w_{y y y}-\frac{3}{2} w^{2} D_{y}\left(\frac{w_{y}^{2}\left(1-c^{2}+c w\right)}{(w-c)^{2}-1}\right) & g_{t}=g_{x x x}+2 g_{x}^{3} \\
\Uparrow & \Uparrow \\
x_{y}=\frac{1}{w}, \quad x_{t}=\frac{1}{2} w_{y}^{2}-w w_{y y}+\frac{3 w_{y}^{2}\left(1-c^{2}+c w\right)}{2\left((w-c)^{2}-1\right)} & y_{x}=c+\sin 2 g, \\
\Downarrow & y_{t}=2 g_{x x} \cos 2 g+2 g_{x}^{2} \sin 2 g \\
\Downarrow & 3\left(y_{x}-c\right) y_{x x}^{2} \\
x_{t}=\frac{1}{x_{y}^{3}}\left(x_{y y y}-\frac{3 x_{y y}^{2}\left(1-3 c x_{y}+2\left(c^{2}-1\right) x_{y}^{2}\right)}{2 x_{y}\left(\left(1-c x_{y}\right)^{2}-x_{y}^{2}\right)}\right) & \Leftrightarrow y_{t}=y_{x x x}-\frac{3}{2\left(\left(y_{x}-c\right)^{2}-1\right)}
\end{array}
$$

По построению уравнение для $w$ должно поддерживать решения типа бризеров. Однако пока неясно, имеет ли оно приложения, и мы не будем его далее рассматривать. 
Построение расширенных одевающих цепочек может основываться на представлении нулевой кривизны

$$
\Phi_{y}=M \Phi, \quad \Phi_{t}=N \Phi \quad \Rightarrow \quad M_{t}=N_{y}+[N, M] .
$$

Так как преобразование Беклунда в случае переменной сепаранты должно менять независимую переменную, то естественно заменить $\partial_{y}, \partial_{t}$ на не зависящие от $n$ производные по параметрам $x, T=t$, положив

$$
\partial_{x}=\rho_{n} \partial_{y_{n}}, \quad \partial_{T}=\partial_{t_{n}}+\sigma_{n} \partial_{y_{n}}, \quad \rho_{n}=y_{n, x}, \quad \sigma_{n}=y_{n, T} .
$$

Тогда условие совместности линейных задач

$$
\Phi_{n, x}=\rho_{n} M_{n} \Phi_{n}, \quad \Phi_{n+1}=L_{n} \Phi_{n}
$$

определит расширенную одевающую цепочку

$$
L_{n, x}=\rho_{n+1} M_{n+1} L_{n}-\rho_{n} L_{n} M_{n}, \quad y_{n, x}=\rho_{n} .
$$

При заданной матрице $M$ отсюда конструктивно находятся как множитель $\rho_{n}$, так и матрица $L_{n}$. Аналогично $t$-часть преобразования Беклунда определяется из условия совместности с линейной задачей

$$
\Phi_{n, T}=\left(N_{n}+\sigma_{n} M_{n}\right) \Phi_{n}, \quad y_{n, T}=\sigma_{n}
$$

Например, уравнение Дима задает изоспектральную деформацию акустического уравнения (5):

$$
\varphi_{y y}=-\lambda w^{-2} \varphi, \quad \varphi_{t}=2 \lambda w_{y} \varphi-4 \lambda w \varphi_{y} .
$$

В матричной форме

$$
\Phi=\left(\begin{array}{c}
\varphi \\
\varphi_{y}
\end{array}\right), \quad M=\left(\begin{array}{cc}
0 & 1 \\
-\lambda w^{-2} & 0
\end{array}\right), \quad N=2 \lambda\left(\begin{array}{cc}
w_{y} & -2 w \\
2 \lambda w^{-1}+w_{y y} & -w_{y}
\end{array}\right) .
$$

Продолжая преобразование Дарбу (8) на $\widehat{\varphi}_{\hat{y}}$, получаем матрицу

$$
L_{n}=\left(\begin{array}{cc}
\gamma_{n} & \left(r_{n+1} r_{n}\right)^{-1} \\
-\lambda r_{n+1} r_{n} & \gamma_{n}
\end{array}\right),
$$

и подстановка $L_{n}, M_{n}=M\left(w_{n}\right)$ в $(28)$ дает уравнения цепочки вместе со связью

$$
\rho_{n}=w_{n}=r_{n}^{-2} \text {. }
$$

Наоборот, нетрудно проверить, что если положить $\operatorname{deg}_{\lambda} L_{n}=\operatorname{deg}_{\lambda} \operatorname{det} L_{n}=1$, то и эта связь, и сама матрица $L_{n}$ однозначно находятся из $(28)$.

ЗАмечАниЕ 2. Имеется гипотеза, что все интегрируемые уравнения вида (24) приводятся дифференциальными подстановками и контактными или точечными преобразованиями к уравнениям с постоянной сепарантой. Если это так, то преобразования Беклунда для уравнений вида (24) можно получить из преобразований 
Беклунда для уравнений (26) при помощи подходящего расширения аналогично приведенным примерам. Интегрируемые уравнения вида (26) изучены очень хорошо, и известно, что все они приводятся (преобразованиями, не меняющими $x$ ) к уравнениям КдФ, Кричевера-Новикова или линейному. Соответственно и все одевающие цепочки сводятся в конечном счете к нескольким базовым. О современном состоянии классификации уравнений (24) см. в работе [13].

\section{5. УРАВНЕНИЕ КАМАССЫ-ХОЛМА}

Уравнение Камассы-Холма [2]

$$
4 h_{t}-h_{z z t}+2 \varepsilon h_{z}=h h_{z z z}+2 h_{z} h_{z z}-12 h h_{z}
$$

возникает как условие совместности линейных задач

$$
\chi_{z z}=\left(1-\lambda R^{4}(z)\right) \chi, \quad \chi_{t}=\frac{h_{z}}{2} \chi+\left(\frac{1}{2 \lambda}-h\right) \chi_{z}, \quad R^{4}:=h_{z z}-4 h-\varepsilon .
$$

Одевающую цепочку для этого уравнения относительно переменных $z, R$ проще всего получить преобразованием Лиувилля (10) из цепочки (21):

$$
z_{n+1, x} z_{n, x}=\gamma_{n}^{2} \operatorname{sh}^{2}\left(z_{n+1}-z_{n}\right), \quad z_{n, x}=R_{n}^{-2} .
$$

Уравнение $(29)$ эквивалентно закону сохранения $\left(R^{2}\right)_{t}+\left(R^{2} h\right)_{z}=0$, что позволяет применить преобразование по решению

$$
d x=R^{2} d z-R^{2} h d t \quad \Rightarrow \quad z_{x}=R^{-2}, \quad z_{t}=h .
$$

Исключая $R$ и $h$ из равенства $R^{4}=\left(z_{x}^{-1} D_{x}\right)^{2}(h)-4 h-\varepsilon$, получаем уравнение

$$
z_{x x t} z_{x}-z_{x t} z_{x x}=\left(4 z_{t}+\varepsilon\right) z_{x}^{3}+z_{x}
$$

являющееся одной из форм записи так называемого ассоциированного уравнения Камассы-Холма [8]. Первое уравнение расширенной цепочки (30) задает $x$-часть преобразования Беклунда для уравнения (31).

Наряду с этим преобразованием уравнение (31) допускает еще одно преобразование Беклунда, которое не содержит параметра и может рассматриваться как предельный случай. Интересным обстоятельством является то, что соответствующая $t$-часть записывается в виде цепочки типа Вольтерра. Чтобы не возникло путаницы, будем обозначать итерации этого преобразования Беклунда верхним индексом.

УТВЕРЖДЕНИЕ 7. Следующая пара цепочек коммутирует:

$$
z_{x}^{m} z_{x}^{m+1}=e^{2 z^{m}-2 z^{m+1}}, \quad-8 z_{t}^{m}=2 \varepsilon+e^{2 z^{m+1}-2 z^{m}}+e^{2 z^{m}-2 z^{m-1}} .
$$

Переменные $z^{m}$ удовлетворяют в силу этих цепочек уравнению (31).

Приведем еще несколько эквивалентных уравнений. Переходя к шредингеровской калибровке, положим $\psi=R \chi$, откуда следует

$$
\psi_{x x}=(u-\lambda) \psi, \quad 2 \lambda \psi_{t}=R^{2} \psi_{x}-R R_{x} \psi, \quad u=\frac{R_{x x}}{R}+\frac{1}{R^{4}}, \quad u_{t}=-2 R R_{x} .
$$


Вводя переменную $v$ по формулам $u=2 v_{x}, R^{2}=-2 v_{t}$ и исключая $R$, приходим к уравнению

$$
2 v_{t} v_{x x t}-v_{x t}^{2}-8 v_{x} v_{t}^{2}+1=0
$$

для которого имеется следующее представление в виде пары совместных цепочек:

$$
v_{x}^{m}+v_{x}^{m+1}=\left(v^{m}-v^{m+1}\right)^{2}, \quad v_{t}^{m}=\left(v^{m+1}-v^{m-1}\right)^{-1} .
$$

Для разностей $F^{m}=v^{m}-v^{m+1}$ получаем пару

$$
F_{x}^{m}+F_{x}^{m+1}=\left(F^{m}\right)^{2}-\left(F^{m+1}\right)^{2}, \quad F_{t}^{m}=\left(F^{m+1}+F^{m}\right)^{-1}-\left(F^{m}+F^{m-1}\right)^{-1} .
$$

Утверждение о ее совместности (а также некоторые другие формы записи) содержится в работах [12], [14], хотя ассоциированное уравнение

$$
F F_{x x t}-F_{x} F_{x t}-4 F^{3} F_{t}+2 F_{x}=0
$$

там выписано не было.

Очевидно, первая из цепочек (33) задает итерации преобразования Дарбу с нулевым параметром (16). Таким образом, данное преобразование Беклунда интерпретируется как продолжение фигуры на рис. 1 в вертикальном направлении, причем в силу (17) выполняется разностное уравнение

$$
\left(v_{n+1}^{m}-v_{n}^{m+1}\right)\left(v_{n}^{m}-v_{n+1}^{m+1}\right)=\alpha_{n} .
$$

Нетрудно проверить, что оно совместно с динамикой по $t$.

\section{6. ЧАСТНЫЕ РЕШЕНИЯ}

Пусть для оператора Шредингера $-D_{x}^{2}+u_{1}$ известны волновые функции $r_{1}=\psi_{1}^{(0)}$ и $\psi_{1}^{\left(\alpha_{n}\right)}$, отвечающие попарно различным значениям спектрального параметра (в случае кратных параметров вид дальнейших формул не меняется, но наряду с волновыми используются присоединенные функции). Применяя преобразование Дарбу, определяемое функцией $\psi_{1}^{\left(\alpha_{1}\right)}$, получим потенциал $u_{2}$, для которого известны волновые функции $\psi_{2}^{\left(\alpha_{n}\right)}=\left(D_{x}-f_{1}\right)\left(\psi_{1}^{\left(\alpha_{n}\right)}\right), n \neq 1$, где $f_{1}=\psi_{1, x}^{\left(\alpha_{1}\right)} / \psi_{1}^{\left(\alpha_{1}\right)}$. Применяя далее преобразования, определяемые функциями $\psi_{2}^{\left(\alpha_{2}\right)}, \psi_{3}^{\left(\alpha_{3}\right)}$ и т.д., построим последовательность кратных преобразований Дарбу. Их результат выражается через вронскианы от исходных функций $\psi_{1}^{\left(\alpha_{n}\right)}$ по формулам Крама [9]:

$$
\begin{gathered}
\psi_{n+1}^{(\lambda)}=\frac{\Delta_{n}\left(\psi_{1}^{(\lambda)}\right)}{\Delta_{n}}, \quad f_{n+1}=D_{x} \ln \left(\frac{\Delta_{n+1}}{\Delta_{n}}\right), \\
u_{n+1}=u_{1}-2 D_{x}^{2} \ln \Delta_{n}, \quad v_{n+1}=v_{1}-D_{x} \ln \Delta_{n}, \\
\Delta_{0}=1, \quad \Delta_{n}=\left\langle\psi_{1}^{\left(\alpha_{1}\right)}, \ldots, \psi_{1}^{\left(\alpha_{n}\right)}\right\rangle, \quad \Delta_{n}(g)=\left\langle\psi_{1}^{\left(\alpha_{1}\right)}, \ldots, \psi_{1}^{\left(\alpha_{n}\right)}, g\right\rangle, \\
\left\langle g_{1}, \ldots, g_{n}\right\rangle:=\left.\operatorname{det}\left(D_{x}^{k-1}\left(g_{j}\right)\right)\right|_{j, k=1} ^{n} .
\end{gathered}
$$

Доказательство следует непосредственно из определения преобразования Дарбу (4) с учетом тождества

$$
\left\langle g_{1}, \ldots, g_{n}\right\rangle=g_{1}\left\langle A\left(g_{2}\right), \ldots, A\left(g_{n}\right)\right\rangle, \quad A=D_{x}-\frac{g_{1, x}}{g_{1}} .
$$


Формулы Крама нетрудно распространить и на другие переменные, введенные в разделе 3 . Так как $r_{1}$ является волновой функцией при $\lambda=0$, то

$$
\bar{v}_{n}=v_{1}-D_{x} \ln \Delta_{n-1}\left(r_{1}\right), \quad F_{n}=D_{x} \ln \left(\frac{\Delta_{n-1}\left(r_{1}\right)}{\Delta_{n-1}}\right) .
$$

Отсюда имеем $r_{n}=c_{n} \Delta_{n-1}\left(r_{1}\right) / \Delta_{n-1}$, а множитель уточним из формулы (19) для $f_{n}$, которую перепишем в виде

$$
\left\langle\Delta_{n}, \Delta_{n-1}\left(r_{1}\right)\right\rangle=\gamma_{n} \frac{c_{n+1}}{c_{n}} \Delta_{n-1} \Delta_{n}\left(r_{1}\right) .
$$

Воспользовавшись тождеством Якоби

$$
\left\langle\left\langle g_{1}, \ldots, g_{n}, g\right),\left\langle g_{1}, \ldots, g_{n}, h\right\rangle\right\rangle=\left\langle g_{1}, \ldots, g_{n}\right\rangle\left\langle g_{1}, \ldots, g_{n}, g, h\right\rangle,
$$

получаем $c_{n+1}=c_{n} / \gamma_{n}=\left(\gamma_{n} \ldots \gamma_{1}\right)^{-1}$. Окончательно имеем следующее параметрическое представление для потенциалов и волновых функций акустического уравнения:

$$
\begin{gathered}
r_{n+1}=\frac{\Delta_{n}\left(r_{1}\right)}{\gamma_{n} \ldots \gamma_{1} \Delta_{n}}, \quad \varphi_{n+1}^{(\lambda)}=\frac{\Delta_{n}\left(\psi_{1}^{(\lambda)}\right)}{\Delta_{n}\left(r_{1}\right)}, \quad y_{n+1}=\sum_{k=1}^{n} \frac{1}{\gamma_{k} r_{k+1} r_{k}}+\int r_{1}^{-2}(\xi) d \xi \\
\bar{r}_{n+1}=\frac{1}{r_{n+1}}, \quad \bar{\varphi}_{n+1}^{(\lambda)}=\frac{\Delta_{n}\left(r_{1}, \psi_{1}^{(\lambda)}\right)}{\Delta_{n}}, \quad \bar{y}_{n+1}=\sum_{k=1}^{n} \frac{r_{k+1} r_{k}}{\gamma_{k}}+\int r_{1}^{2}(\xi) d \xi
\end{gathered}
$$

ПримеР 1. Пусть $u_{1}=c^{2}, \alpha_{n}=c^{2}-\varkappa_{n}^{2}$, где $0<\varkappa_{1}<\cdots<\varkappa_{N-1}<\varkappa_{N}=c$. В качестве волновых функций выберем

$$
\psi_{1}^{\left(\alpha_{n}\right)}=\left\{\begin{array}{ll}
\operatorname{ch}\left(\varkappa_{n} x+\delta_{n}\right), & n=2 k-1, \\
\operatorname{sh}\left(\varkappa_{n} x+\delta_{n}\right), & n=2 k,
\end{array} \quad r_{1}=\psi_{1}^{\left(\alpha_{N}\right)}=\psi_{1}^{(0)} .\right.
$$

Тогда определители $\Delta_{n}$ не обращаются в нуль на вещественной оси и асимптотически $\Delta_{n} \sim e^{\left(\varkappa_{1}+\cdots+\varkappa_{n}\right)|x|}, x \rightarrow \infty$. Так как $\Delta_{N-1}\left(r_{1}\right)=\Delta_{N}$ в силу определения $r_{1}$, то потенциалы $u_{N}=c^{2}-2 D_{x}^{2} \ln \Delta_{N-1}$ и $\bar{u}_{N}=c^{2}-2 D_{x}^{2} \ln \Delta_{N}$ будут обычными многосолитонными потенциалами для оператора Шрединера, приподнятыми на $c^{2}$.

Потенциалы акустической задачи как функции от $x$ имеют асимптотику $r_{N} \sim$ $\operatorname{ch}(c x), \bar{r}_{N} \sim \operatorname{sech}(c x)$. Они, а также соответствующие аргументы $y_{N}, \bar{y}_{N}$, изображены на рис. $2 \mathrm{a}, 2$ г. Функции $y_{N}, \bar{y}_{N}$ строго монотонно возрастают, но $y_{N}$ ограничена, а $\bar{y}_{N}$ растет на бесконечности как $\operatorname{sh}(2 c x)$. Рассмотрим эти случаи отдельно.

1. Выберем в представлении (34) значение первообразной, равное $c^{-1} \operatorname{th}(c x+$ $\left.\delta_{N}\right)$ при $N=2 k-1$ и $c^{-1} \operatorname{cth}\left(c x+\delta_{N}\right)$ при $N=2 k$. Тогда легко показать, что масштабированная переменная $y=c y_{N}$ меняется от -1 до 1 , и график $w(y)=c r_{N}^{-2}$ имеет вид финитной шапочки (рис. 2б). Зависимость фаз от $t$ вида

$$
\delta_{n}=\varkappa_{n}\left(4 \varkappa_{n}^{2}-6 c^{2}\right) t+\tilde{\delta}_{n}, \quad \tilde{\delta}_{n}=\mathrm{const},
$$

приводит к решению уравнения Дима (27) на отрезке $[-1,1]$ с нулевыми граничными условиями. 


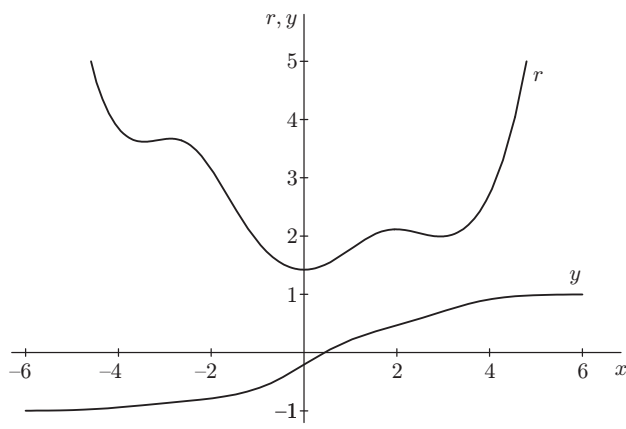

a
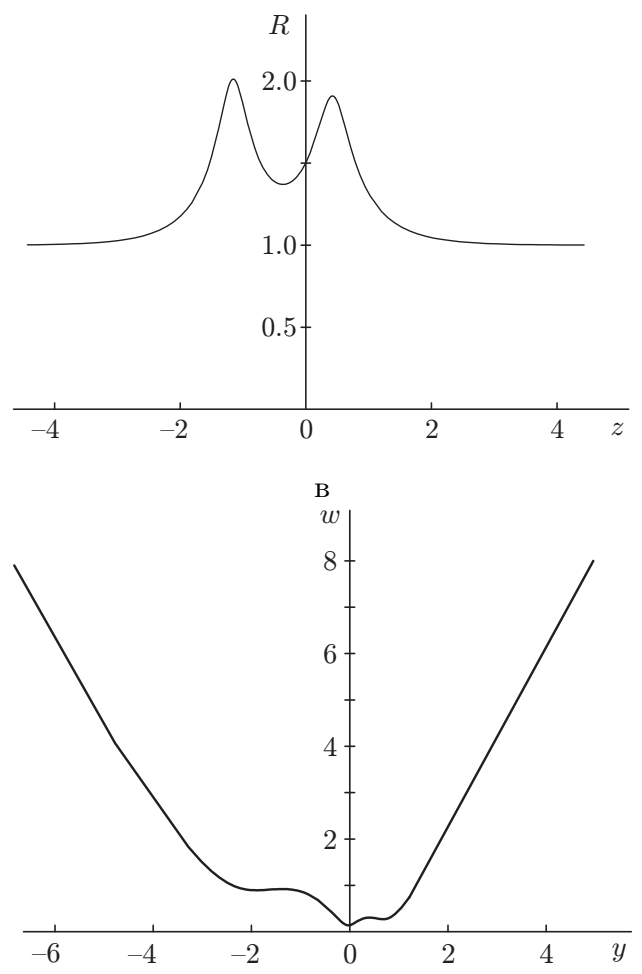

Д

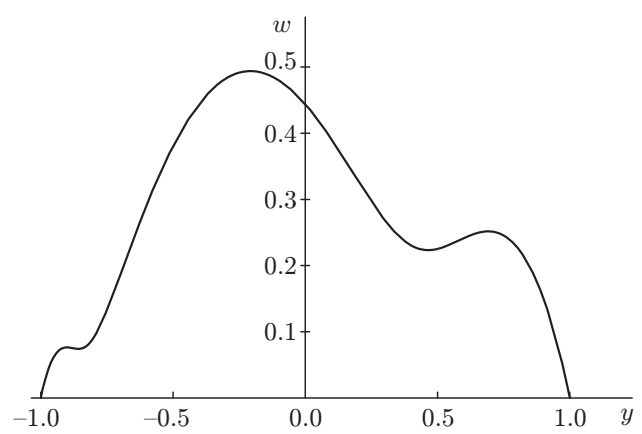

б

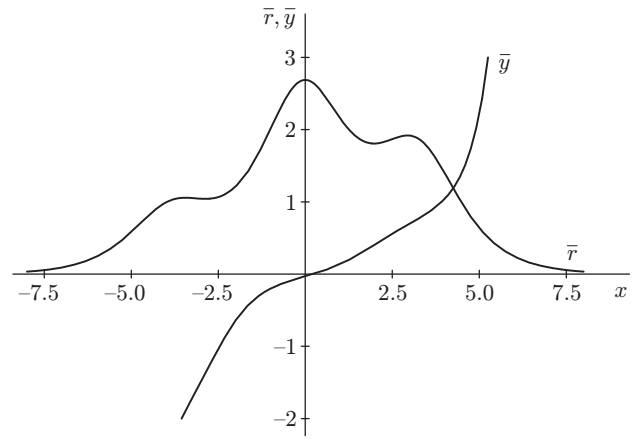

$\Gamma$

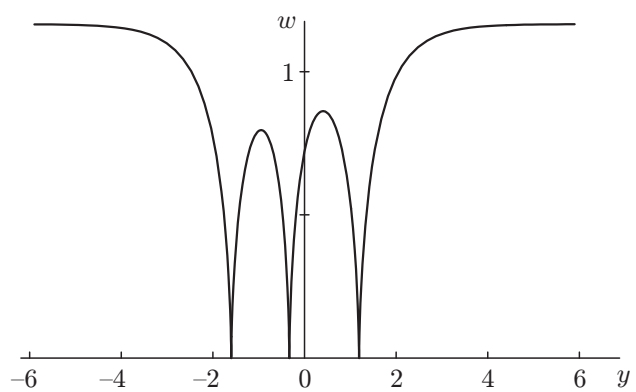

e

Рис. 2. Трехсолитонный потенциал в разных координатах (а-д); потенциал с особенностями (е).

Применяя теперь преобразование Лиувилля (10), получаем функцию $R(z)$ в параметрическом виде (рис. 2в)

$$
R=r_{N}(x) \sqrt{1-y^{2}(x)}, \quad z=\frac{1}{2} \ln \frac{1+y(x)}{1-y(x)} .
$$


При этом один солитон уничтожается преобразованием. Можно показать, что зависимость фаз от $t$, отвечающая уравнению Камассы-Холма, имеет вид

$$
\delta_{n}=\frac{\varkappa_{n} t}{2 c\left(c^{2}-\varkappa_{n}^{2}\right)}+\tilde{\delta}_{n}, \quad n=1, \ldots, N-1, \quad \delta_{N}=-\frac{\left(1+\varepsilon c^{2}\right) t}{4 c^{2}}+\tilde{\delta}_{N} .
$$

2. Функция $w(y)=r_{N}^{2}, y=\bar{y}_{N}$, асимптотически имеет линейный рост (рис. 2 д). Она задает решение уравнения Дима, если фазы $\delta_{n}$ зависят от $t$ согласно формулам (36), а в качестве первообразной принято

$$
\bar{y}_{1}=\frac{1}{4 c} \operatorname{sh} 2 \delta_{N}+(-1)^{N-1}\left(\frac{x}{2}+3 c^{2} t\right)+\text { const } .
$$

ПримеР 2. Пусть $u_{1}=0, \alpha_{n}=-\gamma_{n}^{2}$, где $\gamma_{N}=0<\gamma_{1}<\cdots<\gamma_{N-1}$,

$$
\psi_{1}^{\left(\alpha_{n}\right)}=\left\{\begin{array}{ll}
\operatorname{ch}\left(\gamma_{n} x+4 \gamma_{n}^{3} t+\delta_{n}\right), & n=2 k-1, \\
\operatorname{sh}\left(\gamma_{n} x+4 \gamma_{n}^{3} t+\delta_{n}\right), & n=2 k,
\end{array} \quad r_{1}=\psi_{1}^{(0)}=1 .\right.
$$

Тогда функция $r_{N}(x)=\Delta_{N-1}(1) / \Delta_{N-1}$ имеет $N-1$ нуль. Полагая $\bar{y}_{1}=x$, получим по формулам $w(y)=r_{N}^{2}, y=\bar{y}_{N}$, решение уравнения Дима с $N-1$ особенностью (рис. 2e).

\section{7. ЗАКЛЮЧЕНИЕ}

Основной вывод статьи можно сформулировать так: цепочку преобразований Беклунда для уравнения с переменной сепарантой удобно записывать в параметрическом виде как двухкомпонентную цепочку для зависимой и независимой переменных. Мы проиллюстрировали это на примере одевающей цепочки для уравнения Дима, являющейся расширением цепочки для модифицированного уравнения КдФ. Она порождается преобразованием Дарбу для оператора Шредингера в акустической калибровке в отличие от одевающей цепочки для уравнения КдФ, отвечающей стандартной калибровке. Оба варианта объединяются на дважды дискретном уровне. Уравнение Камассы-Холма также укладывается в общую схему.

За рамками нашей работы осталось уравнение sin-Гордон, с которым связано, например, уравнение сверхкоротких импульсов [15]. Возможно, замечание 1 указывает способ построения преобразования Беклунда и в этом случае, но, скорее всего, удобнее перейти к спектральной задаче Захарова-Шабата. С этой задачей ассоциированы также двухкомпонентные аналоги уравнений Дима и Камассы-Холма, привлекшие внимание исследователей лишь недавно (см., например, [16]). По сравнению с оператором Шредингера здесь имеется большее разнообразие преобразований Дарбу, порождающих, в частности, цепочки типа Тоды и релятивистской цепочки Тоды.

Интерес представляет также найденное недавно уравнение Дегаспериса-Прокеси [17], весьма близкое уравнению Камассы-Холма, но ассоциированное со спектральной задачей Каупа-Купершмидта, имеющей третий порядок. Это делает реализацию общей схемы достаточно трудной, так как преобразование Дарбу для этой задачи более громоздко (см., например, [18]). 
По-видимому, не представляет принципиальных трудностей построение расширенных цепочек преобразований Дарбу и Лапласа, обслуживающих $(2+1)$-мерные уравнения с переменной сепарантой. Типичным примером является обобщение уравнения Дима, изучавшееся в работах [19].

Благодарности. Авторы благодарят В. В. Соколова и М. В. Павлова за полезные обсуждения. Работа выполнена при поддержке гранта РФФИ № 04-01-00403.

\section{Список литературы}

[1] M. Kruskal, "Nonlinear wave equations", Dynamical systems, theory and applications, Lect. Notes in Phys., 38, ed. J. Moser, Springer, Heidelberg, 1975, 310-354.

[2] B. Fuchssteiner, A.S. Fokas, Physica D, 4 (1981), 47-66; R. Camassa, D. D. Holm, Phys. Rev. Lett., 71 (1993), 1661-1664.

[3] М.Г. Крейн, ДАН СССР, 82 (1952), 669-672.

[4] F. Calogero, A. Degasperis, Spectral transforms and solitons, North-Holland, Amsterdam, 1982.

[5] R. Beals, D. Sattinger, J. Szmigielski, Adv. Math., 140 (1998), 190-206.

[6] A. Constantin, Proc. Roy. Soc. Lond. A, 457 (2001), 953-970.

[7] L. A. Dmitrieva, Phys. Lett. A, 182:1 (1993), 65-70; J. Phys. A, 26 (1993), 6005-6020; A. Constantin, H.P. McKean, Commun. Pure Appl. Math., 52 (1999), 949-982; В. С. Новиков, Писъма в ЖЭЭТФ, 72:3 (2000), 223-228; М. S. Alber, Yu. N. Fedorov, Inverse Problems, 17 (2001), 1017-1042; M. S. Alber, R. Camassa, Yu. N. Fedorov, D. D. Holm, J.E. Marsden, Commun. Math. Phys., 221 (2001), 197-227; R. Camassa, A. I. Zenchuk, Phys. Lett. A, 281 (2001), 26-33.

[8] J. Schiff, Physica D, 121 (1998), 24-43; A. N.W. Hone, J. Phys. A, 32 (1999), L307-L314; R. Ivanov, Phys. Lett. A, 345 (2005), 112-118; nlin.SI/0507005.

[9] M. M. Crum, Quart. J. Math. Oxford 2, 6 (1995), 121-127.

[10] Дж. Л. Лэм, Введение в теорию солитонов, Мир, М., 1984.

[11] А. В. Shabat, Inverse Problems, 8 (1992), 303-308; А. П. Веселов, А. Б. Шабат, Функи. анализ и его прилож., 27:2 (1993), 1-21.

[12] А.Б. Шабат, Р.И. Ямилов, Алгебра и анализ, 2:2 (1990), 183-208.

[13] R. H. Heredero, J. Nonlinear Math. Phys., 12:4 (2005), 567-585.

[14] Р. И. Ямилов, ТМФ, 85:3 (1990), 368-375.

[15] R. Beals, M. Rabelo, K. Tenenblat, Stud. Appl. Math., 81 (1989), 125-151; T. Schäfer, C. E. Wayne, Physica D, 196 (2004), 90-105.

[16] G. Falqui, On a Camassa-Holm type equation with two dependent variables, nlin.SI/0505059; H. Aratyn, J.F. Gomes, A.H. Zimerman, J. Phys. A, 39 (2006), 1099-1114.

[17] A. Degasperis, M. Procesi, "Asymptotic integrability", Symmetry and perturbation theory, Proc. 2nd Int. Workshop (Rome, Italy, December 16-22, 1998), eds. A. Degasperis, G. Gaeta, World Scientific, Singapore, 1999, 23-37; А. Дегасперис, Д.Д. Холм, А.НИ. Хон, ТМФ, 133:2 (2002), 170-183.

[18] В.Э. Адлер, В.Г. Марихин, А.Б Шабат, ТМФ, 129:2 (2001), 163-183.

[19] V. G. Dubrovsky, B. G. Konopelchenko, J. Phys. A, 27:13 (1994), 4619-4628; L. A. Dmitrieva, M. A. Khlabystova, Phys. Lett. A, 237:6 (1998), 369-380. 